\title{
Chest Diameter Measurement in Pediatric Patients for Chest Compression Feedback Calibration
}

\author{
Sofía Ruiz de Gauna ${ }^{1}$, Digna M González-Otero ${ }^{1}$, Jesus Ruiz ${ }^{1}$, Stefano de Nigris ${ }^{1,2}$, \\ Purificación Saiz ${ }^{1}$, JJ Gutiérrez ${ }^{1}$, James K Russell ${ }^{3}$, Elena De Momi ${ }^{2}$ \\ ${ }^{1}$ University of the Basque Country (UPV/EHU), Bilbao, Spain \\ ${ }^{2}$ Politecnico di Milano, Milan, Italy \\ ${ }^{3}$ Oregon Health and Science University (OHSU), Portland, OR, USA
}

\begin{abstract}
Adequate compression depth is a main quality parameter during cardiopulmonary resuscitation (CPR). Current CPR feedback devices can guide adult target depth which is fixed between 5 and $6 \mathrm{~cm}$. For pediatric patients, conversely, target depth should be one third of the antero-posterior diameter of the chest. The aim of this study was to develop an algorithm to estimate chest diameter in pediatric patients using accelerometers.

Using a tri-axial accelerometer, we measured the accelerations generated when moving the sensor from the floor to five different heights that simulated chest diameter. Five volunteers generated two records each per height. A total of fifty records were acquired. Chest diameter was measured by discrete integration of the zaxis acceleration signal. Velocity signal was band-pass filtered before computing the displacement signal. Chest diameter was identified as the displacement value at the instant in which the movement finished.

Median $\left(P_{25}, P_{75}\right)$ unsigned absolute and relative errors were $0.9 \mathrm{~cm}(0.3,1.9)$ and $9.2 \%(2.5,14.6)$, respectively. Error in estimation of pediatric target compression depth was below $6.5 \mathrm{~mm}$ in $75 \%$ of the cases.

The proposed algorithm could be used to calibrate target chest compression depth in CPR feedback devices to be adapted for pediatric patients.
\end{abstract}

\section{Introduction}

During cardiopulmonary resuscitation (CPR), rescuers should provide high-quality chest compressions to the victim to maximize survival of cardiac arrest [1]. The objective of chest compressions is to provide a minimal blood flow to the vital organs. However, studies on CPR quality showed that delivering adequate chest compressions was difficult even for well-trained rescuers both in hospital and out of hospital [2,3]. Pauses between compressions were very frequent, compressions were often too shallow, and there was a tendency to provide too fast chest compressions.

Recent development of feedback devices, capable of real-time monitoring and guidance of chest compressions has contributed to improve CPR quality [4,5]. These devices are usually placed between the chest of the patient and the rescuer's hands, and guide rescuers towards the target compression depth and rate. Many widespread devices are based on accelerometer sensors.

For adult patients, target compression depth is fixed between 5 and $6 \mathrm{~cm} \mathrm{[6].} \mathrm{For} \mathrm{pediatric} \mathrm{patients,}$ conversely, target depth is one third of the anteroposterior diameter of the chest, and thus varies depending on patient age and morphology [7]. Available feedback devices are designed for adults and there is no available solution adapted to pediatrics.

The aim of this study was to develop an algorithm to estimate chest diameter in pediatric patients using one accelerometer.

\section{Materials and methods}

\subsection{Data collection}

We used an analog tri-axial accelerometer (ADXL330, Analog Devices, USA) encased in a metal box to record the accelerations generated when moving the sensor from the floor to five different heights which simulated chest diameter. Target heights were $8,10,12,14$, and $16 \mathrm{~cm}$ (which corresponded to compression depths of 2.7, 3.4, 4, 4.7, and $5.4 \mathrm{~cm}$ ).

Five volunteers participated in the experiments. Each volunteer did the floor-to-height movement twice for each target. Figure 1 shows a representation of the tested movements. First, the initial point was closer to the target (see Figure 1, distance $d_{1}$ ), and second, the initial point was more distant to the target (Figure 1, distance $d_{2}$ ). We collected a total of 50 records with the three axis accelerations, sampled at a rate of 1000 samples/s and stored with a 16 bit resolution. 


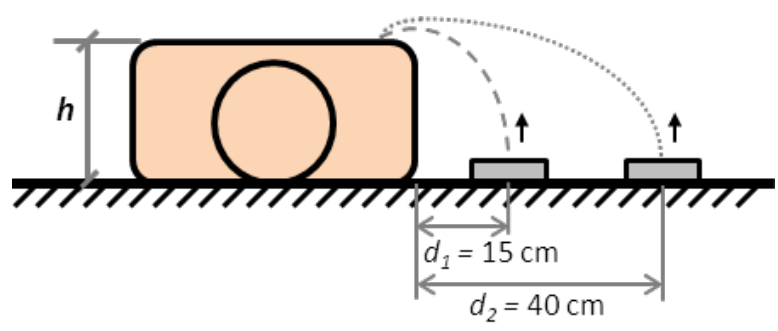

Figure 1. Representation of the experiment.

\subsection{Estimation of the chest diameter}

Chest diameter (height $h$, see Figure 1) was estimated by applying double integration to the z-axis (perpendicular to the floor) acceleration. Discrete integration was approximated by the trapezoidal rule, which was implemented as a linear filter with the following transfer function $H(\mathrm{z})$, where $T_{S}$ is the sampling period:

$$
H(z)=\frac{T_{s}}{2} \cdot \frac{1+z^{-1}}{1-z^{-1}}=\frac{T_{s}}{2} \cdot \frac{z+1}{z-1}
$$

First, acceleration signal was calibrated and gravity was then subtracted from the acceleration. The integration filter was applied once to the acceleration to obtain the velocity signal, and once again to obtain the displacement signal. As the filter was unstable (pole in $z=1$ ) error accumulation made this approximation of the trapezoidal rule unfeasible [8]. An example of this effect is shown in Figure 2, where the filter was directly applied to the acceleration signal (top panel) corresponding to a record with a target $h$ of $14 \mathrm{~cm}$. The accumulation error can be observed in the velocity signal after the first filtering step (middle panel). Moreover, integration errors produced extremely high errors in displacement (bottom panel). Movement was initiated at $3 \mathrm{~s}$, and finished $2 \mathrm{~s}$ later approximately. At $4.5 \mathrm{~s}$, the estimated $h$ was $68.8 \mathrm{~cm}$.

In order to reduce the accumulation of integration errors, we established boundary conditions to velocity. In the chest diameter calibration movement, the initial and final velocities can be considered equal to zero, so we imposed the condition that the DC component of the acceleration is null. Figure 3 shows, for the same record, how integration errors decreased in the calculation of velocity and displacement signals. However, chest diameter $h$ was again overestimated, as measured value was $29.6 \mathrm{~cm}$. The main reason of these high errors is that, although the initial velocity is exactly zero (the movement starts in the floor), the final velocity at the moment the sensor reaches the target height is not zero, making the initial assumption for the boundary conditions not valid.
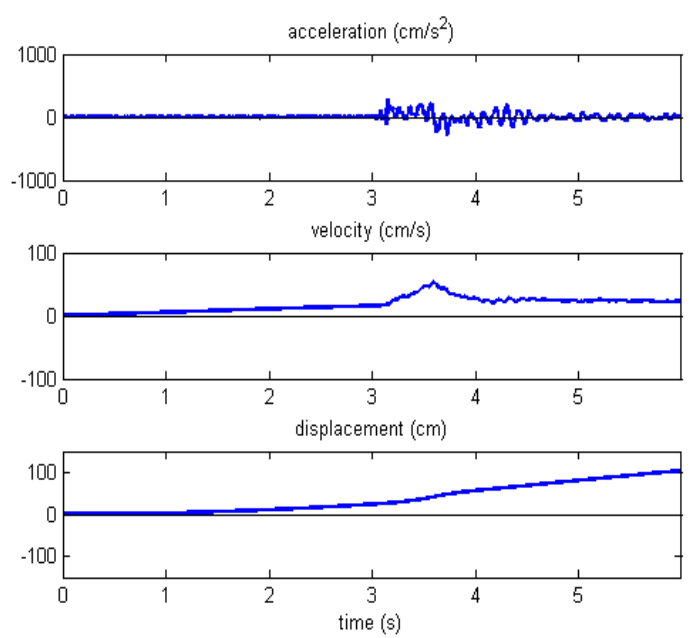

Figure 2. Integration errors after double integration with the trapezoidal rule implemented as a linear system. Estimated $h$ was $68.8 \mathrm{~cm}$ (at $4.5 \mathrm{~s}$ ), target $h$ was $14 \mathrm{~cm}$.
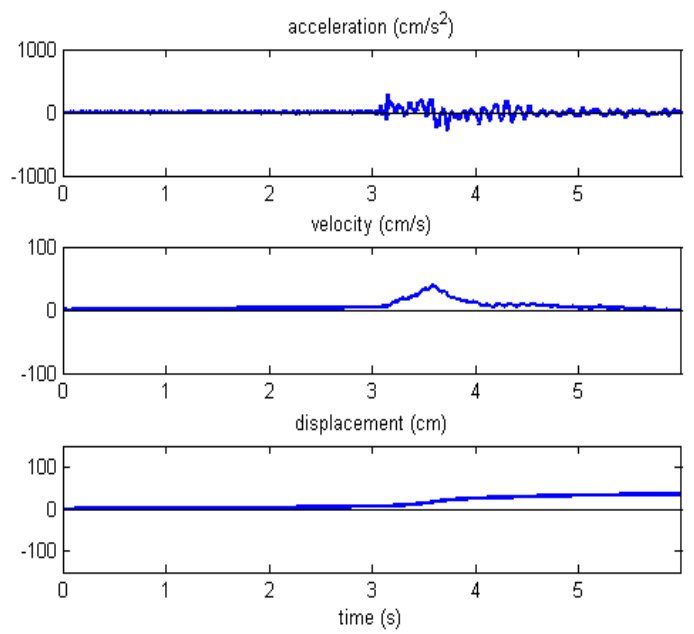

Figure 3. Integration errors after double integration with boundary conditions imposed to velocity. Estimated $h$ was $29.6 \mathrm{~cm}$ (at $4.5 \mathrm{~s}$ ), while target $h$ was $14 \mathrm{~cm}$.

After these initial trials, we improved the chest diameter estimation algorithm to minimize the integration errors. The main steps of the final algorithm are illustrated in Figure 4, and can be described as follows:

1. Movement-onset detection: a threshold th is applied to the z-axis acceleration signal (in $g$ units) to estimate the starting time of the movement, $t_{i}$, as the instant when the acceleration exceeds $t h$.

2. Calibration: The acceleration interval previous to $t_{i}$ is used to calibrate acceleration and then to subtract gravity. The resulting acceleration is depicted in Figure 4, top panel. 
3. Velocity computation: Trapezoidal rule filter is applied to the calibrated acceleration from the instant $t_{i}$ to the end of the record to obtain the velocity signal (Figure 4, second panel).

4. Velocity conditioning: Velocity is low pass filtered with a $3 \mathrm{~dB}$ cut-off frequency of $6 \mathrm{~Hz}$ (Butterworth, $3^{\text {rd }}$ order). The resulting signal is depicted in Figure 4, third panel.

5. Movement ending detection: The instant when the movement finished, $t_{e}$, is identified as the first zero-crossing instant or as the first minimum of the velocity signal after $t_{i}$ if no zero-crossing value is identified.

6. Displacement computation: Displacement signal is obtained by applying the trapezoidal rule filter to the velocity from the instant $t_{i}$ to the end of the record (Figure 4, bottom panel).

7. Chest diameter estimation: Estimated $h$ is identified with the value of the displacement signal at instant $t_{e}$ (marked with a cross in the bottom panel of Figure 4).
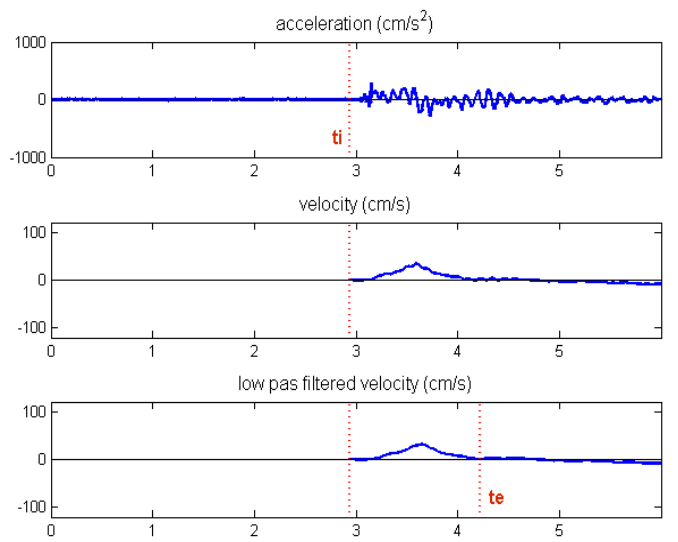

Estimated displacement (cm) $h=14.4 \mathrm{~cm}$

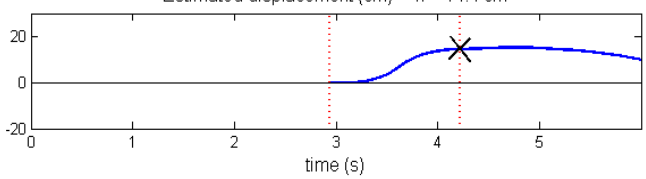

Figure 4. Graphical description of the algorithm. Target $h$ was $14 \mathrm{~cm}$, and estimated $h$ was $14.4 \mathrm{~cm}$ (marked with a red cross in the bottom panel).

\section{Results}

Threshold th to detect the movement onset $t_{i}$ in the zaxis acceleration (step 1 of the algorithm) was fixed to 1.1 g. The algorithm was then applied to the 50 records of the dataset and $h$ was computed for each record. The error in the estimation of chest diameter was computed as the difference between estimated $h$ and actual $h$. Figure 5 shows the distribution of the error for the whole dataset and as a function of the starting position (see Figure 1).
Median $\left(\mathrm{P}_{25}, \mathrm{P}_{75}\right)$ global error was $-0.55 \mathrm{~cm}(-1.8,0)$. Measurements at the farther distance $\left(d_{2}=40 \mathrm{~cm}\right)$ had lower bias and variance than at the nearer distance $\left(d_{1}=15 \mathrm{~cm}\right)$.
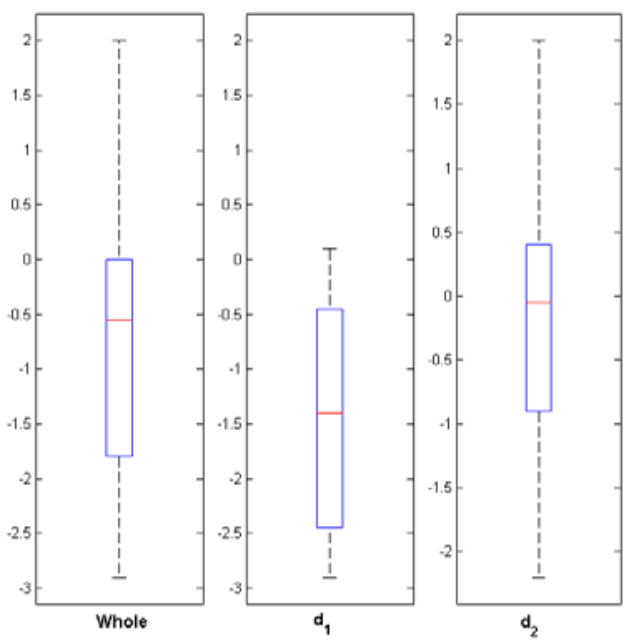

Figure 5. Distribution of the error (in $\mathrm{cm}$ ) in the estimation of chest diameter, for the whole set, and for measurements at $d_{1}(15 \mathrm{~cm})$ and $d_{2}(40 \mathrm{~cm})$.

Median $\left(\mathrm{P}_{25}, \mathrm{P}_{75}\right)$ global unsigned error was $0.9 \mathrm{~cm}$ $(0.3,1.9)$. This corresponded to a percent error of $9.2 \%$ $(2.5,14.6)$. Error in adequate chest compression depth estimation was therefore below $6.5 \mathrm{~mm}$ in $75 \%$ of the cases.

\section{Discussion}

Monitoring CPR performance in resuscitation attempts has been pivotal in resuscitation science [1]. The use of CPR feedback devices has increased chest compression quality, minimizing pauses and increasing compression depth in adults [4]. Current devices are designed for adult patients, for whom a fixed compression depth range is recommended. Adult guidelines recommend a uniform depth range between 5 and $6 \mathrm{~cm}$. Current pediatric recommendation for compression depth is at least one third the anterior-posterior diameter of the chest [7]. This variable target complicates rescuer's intervention in pediatric patients. First, commercial CPR feedback devices are not prepared to be used in this scenario, and second, estimation of target chest compression depth relies on a subjective in-situ measurement of the chest diameter by the rescuers.

An intermediate solution could be to allow rescuers to type this subjective value in the feedback device (standalone) or in the monitor-defibrillator (connected devices) prior to initiate chest compressions, but this would be time consuming and add complexity to the device. 
Many commercial devices are based on accelerometers, so this work has analyzed the feasibility of an automatic estimation of the chest diameter using the acceleration signal recorded by the feedback device. Before positioning the sensor on the chest to initiate chest compressions, a previous calibration action would be required: the rescuer should be instructed to move the sensor from the floor to the patient's chest.

Our algorithm performed accurately for the tested heights, with a median unsigned error below $1 \mathrm{~cm}$, which would support an error in the estimation of the target compression depth below $4 \mathrm{~mm}$. Implementation in current accelerometer-based devices could be straightforward. An important consideration is that during the calibration process, special care is needed to maintain the sensor perpendicular to the floor in order to ensure an accurate measurement. This may explain differences with respect to the initial position of the sensor.

Consensus on resuscitation science is suggesting that CPR performance should be adapted to each patient [1]. This method could guide chest compression depth could be tailored individually to patient's chest size in adults as well, if desired.

In conclusion, this work presented an accurate solution for the automatic estimation of patient's chest diameter based on accelerometers. It could be directly implemented in current devices to adapt them for pediatric use.

\section{Acknowledgements}

This work received financial support from the Spanish Government (project TEC2012-31144), and from the Vice Chancellor's office for Research of the University of the Basque Country, UPV/EHU (grant "Contratación de doctores recientes hasta su integración en programas de formación postdoctoral”).

The authors would like to thank all colleagues participating in collecting the data.

\section{References}

[1] Meaney PA, Bobrow BJ, Mancini ME, et al. Cardiopulmonary Resuscitation Quality: Improving Cardiac Resuscitation Outcomes Both Inside and Outside the Hospital: A Consensus Statement From the American Heart Association. Circulation 2013;128:417-35.

[2] Abella BS, Alvarado JP, Myklebust H, et al. Quality of cardiopulmonary resuscitation during in-hospital cardiac arrest. JAMA: the Journal of the American Medical Association 2005;293; 305-10.

[3] Wik L, Kramer-Johansen J, Myklebust H, et al. Quality of cardiopulmonary resuscitation during out-of-hospital cardiac arrest. JAMA: the Journal of the American Medical Association 2005;293; 299-04.

[4] Stiell IG, Brown SP, Christenson J, et al. Resuscitation Outcomes Consortium Investigators. What is the role of chest compression depth during out-of-hospital cardiac arrest resuscitation? Crit Care Med 2012;40:1192-8.

[5] Gruber J, Stumpf D, Zapletal B, Neuhold S, and Fischer H. Real-time feedback systems in CPR. Trends in Anaesthesia and Critical Care 2012; 2; 287-94.

[6] Monsieurs KG, Nolan JP, Bossaert LL, et al. European Resuscitation Council Guidelines for Resuscitation 2015 Section 1. Executive summary. Resuscitation 2015;95:1-80.

[7] Maconochie IK, Bingham R, Eich C, et al. European Resuscitation Council Guidelines for Resuscitation 2015 Section 6. Paediatric life support. Resuscitation 2015;95:223-48.

[8] Ruiz de Gauna S, González-Otero DM, Ruiz J, Russell JK. Feedback on the Rate and Depth of Chest Compressions during Cardiopulmonary Resuscitation Using Only Accelerometers. PLoS ONE 2016;11:e0150139.

Address for correspondence.

Sofía Ruiz de Gauna

Department of Communications Engineering

Faculty of Engineering

Alameda Urquijo S/N, 48013-Bilbao (Spain)

sofia.ruizdegauna@ehu.es 